\title{
Principais dificuldades do ensino remoto e uso de tecnologias no ensino- aprendizagem de estudantes de Educação Física durante a pandemia da COVID-19
}

\author{
Main difficulties of remote teaching and the use of technologies in the teaching-learning of Physical Education \\ students during the COVID-19 pandemic
}

Principales dificultades de la enseñanza a distancia y el uso de tecnologías en la enseñanza-aprendizaje de estudiantes de Educación Física durante la pandemia COVID-19

\author{
Juliana Daniele de Araújo Silva \\ Universidade Federal de Pernambuco (UFPE), Brasil \\ julianadanielearaujo@gmail.com \\ (iD https://orcid.org/0000-0002-3610-0754
}

Júlia Carolina Lopes Silva

Universidade Federal de Pernambuco (UFPE), Brasil

julia.lopes@ufpe.br

iD https://orcid.org/0000-0002-9682-6581

\author{
Diógenes Cândido Mendes Maranbäo Universidade \\ Federal de Pernambuco (UFPE), Brasil \\ diogesmendes@gmail.com \\ (iD https://orcid.org/0000-0002-4320-0227
}

\author{
Tereza Luiza de França \\ Universidade Federal de Pernambuco (UFPE), Brasil \\ tereza.franca@ufpe.br \\ https://orcid.org/0000-0003-4168-2258
}

\begin{abstract}
Resumo:
O objetivo desta pesquisa foi o de identificar e discutir as principais dificuldades do ensino remoto e uso de tecnologias, em face à pandemia da COVID-19, no ensino-aprendizagem do curso superior de Educação Física através de críticas apontadas por graduandos. Nesta pesquisa descritiva quali-quantitativa feita no segundo semestre de 2020, através de um questionário on-line, foram coletadas as percepções de 26 estudantes sobre os principais pontos negativos vivenciados e/ou identificados no primeiro semestre de implementação do ensino remoto. Posteriormente, foi feita uma análise de conteúdo do material. Foi possível concluir que os principais problemas do ensino remoto e uso de tecnologias durante a pandemia da COVID-19 no ensino-aprendizagem de Educação Física são as metodologias de avaliação insatisfatórias, problemas com o acesso à internet e recursos tecnológicos, dificuldades de aprendizagem, prejuízos na qualidade do ensino, e a ausência de aulas práticas e o aumento do comportamento sedentário.
\end{abstract}

Palavras-CHAVE: Aprendizagem, Educação superior, Ensino online.

\section{Abstract:}

The objective of this research was to identify and discuss the main difficulties of remote teaching and the use of technologies, in view of the COVID-19 pandemic, in the Physical Education course teaching-learning process through criticisms pointed out by undergraduate students. In this qualitative and quantitative descriptive research carried out in the second semester of 
2020, through an online questionnaire, the perceptions of 26 students about the main negative points experienced and/or identified in the first semester of remote learning implementation were collected. Subsequently, a content analysis of the material was performed. It was possible to conclude that the main problems of remote learning and the use of technologies during the COVID-19 pandemic in Physical Education teaching-learning process are the unsatisfactory assessment methodologies, problems with access to the Internet and technological resources, learning difficulties, impairments in the quality of teaching, the absence of practical classes and the increase in sedentary behavior.

KEYWORDS: Apprenticeship, University education, Online teaching.

\section{Resumen:}

El objetivo de esta investigación fue identificar y discutir las principales dificultades de la enseñanza a distancia y el uso de tecnologías, ante la pandemia COVID-19, en la enseñanza-aprendizaje del curso de Educación Física a través de críticas señaladas por estudiantes de pregrado. En esta investigación descriptiva cualitativa y cuantitativa realizada en el segundo semestre de 2020 , a través de un cuestionario online, se recogieron las percepciones de 26 estudiantes sobre los principales puntos negativos vividos y/o identificados en el primer semestre de implementación del aprendizaje a distancia. Posteriormente, se realizó un análisis de contenido del material. Se pudo concluir que los principales problemas del aprendizaje remoto y el uso de tecnologías durante la pandemia COVID-19 en la enseñanza-aprendizaje de la Educación Física son las metodologías de evaluación insatisfactorias, problemas de acceso a internet y recursos tecnológicos, dificultades de aprendizaje, pérdidas en la calidad de la enseñanza, y la ausencia de clases prácticas y el aumento del sedentarismo.

Palabras clave: Aprendizaje, Educación universitária, Enseñanza online.

\section{INTRODUÇÃO}

Desde o início de março de 2020 a sociedade enfrenta a pandemia da COVID-19, uma das doenças causadas pelo coronavírus, uma família de agentes virais que causam infecções respiratórias (Alves \& Ferreira, 2020). Diante do cenário pandêmico, várias medidas foram adotadas para evitar o aumento exponencial de casos de COVID-19, pautadas principalmente no isolamento social, como a restrição de viagens e circulação de pessoas, proibição de aglomerações, e o fechamento de estabelecimentos como cinemas, restaurantes, academias e locais de culto (Aquino et al., 2020).

Devido ao fechamento das escolas e instituições, a educação brasileira desde o nível básico ao superior de ensino também foi alvo das implementações de medidas contra os impactos do coronavírus, através da realização massiva de propostas de educação à distância (de França Filho, da França Antunes, Couto, 2020). Especificamente sobre as instituições de ensino superior, várias aderiram ao Ensino Remoto Emergencial, um sistema educacional não robusto, caracterizado como um modo de ensino alternativo devido às circunstâncias de crise, que envolve o uso de soluções de ensino totalmente remotas para permitir acesso temporário aos conteúdos programáticos que seriam ofertados de forma presencial (Hodges et al., 2020).

O ensino remoto vem ganhando protagonismo nos momentos de crise pela pandemia da COVID-19, onde os professores assumiram os desafios de construir novas formas de ensinar e de aprender, ressignificando práticas pedagógicas que precisam ser adequadas para o novo modelo de ensino (Valente et al., 2020). Essa transição de forma abrupta não é algo simples de ser feita, em especial no campo da Educação Física, como Godoi et al. (2021) relataram ao falar sobre os desafios profissionais relacionados ao ensino remoto no contexto de isolamento social, onde professores de Educação Física tiveram que aprender a utilizar novas ferramentas e desenvolver novas estratégias de ensino e habilidades de trabalho e emocionais.

Precisamente, os professores de Educação Física tiveram que transferir suas aulas das quadras esportivas, campos e piscinas para a frente das telas do computador ou do celular (Godoi et al., 2021), porém, apesar de o trabalho com tecnologias e ambientes virtuais e da discussão da necessidade do conhecimento e metodologias específicas dos professores sobre o papel das tecnologias nas aulas de Educação Física serem discutidos muito antes do isolamento social (Fernando, 2017), grande parte dos docentes não possuem formação que os capacitem para o modelo remoto de ensino, aumentando as dificuldades pedagógicas (Có, Amorim, Finardi, 2020). 
Diante do novo cenário de educação na pandemia da COVID-19 e suas incertezas tanto no presente quanto na fase pós-pandemia, é importante investigar como estão acontecendo os processos pedagógicodidáticos no campo da Educação Física e, principalmente, identificar os principais problemas envolvidos no processo de ensino-aprendizagem no modelo remoto de ensino. Tais achados podem contribuir para que sejam estruturados planos educacionais de forma a permitir uma melhor formação acadêmica em relação à Educação Física, maximizando o uso de tecnologias de forma facilitadora, bem como a inclusão social e à educação dos corpos. Assim, a presente pesquisa visa identificar e discutir as principais dificuldades do ensino remoto e uso de tecnologias, em face à pandemia da COVID-19, no ensino-aprendizagem do curso superior de Educação Física através de críticas apontadas por graduandos.

\section{MÉToDos}

O presente artigo trata-se de uma pesquisa descritiva de natureza quantitativa. Foi feita uma pesquisa de levantamento, caracterizada pelo interesse de proporcionar uma descrição quantitativa ou numérica de tendências, de atitudes ou de opiniões de uma população, utilizando questionários ou entrevistas estruturadas para coletas de dados, estudando uma amostra da população de interesse (Creswell, 2010).

A amostra foi composta por 26 estudantes concluintes de graduação em Bacharelado Educação Física da Universidade Federal de Pernambuco, Brasil, cursantes da disciplina de Trabalho de Conclusão de curso (TCC), mediante a uma consulta à comunidade acadêmica autorizada pelo Departamento. A pesquisa foi realizada durante o segundo semestre do ano de implementação do Ensino Remoto Emergencial, em 2020, sendo o semestre exclusivamente realizado no modelo remoto de ensino. As coletas foram feitas de forma remota, tendo como instrumento um questionário, uma ferramenta usada para coletar dados por meio de perguntas a respeito de uma ou mais variáveis que serão mensuradas (Sampieri, Collado, Lucio, 2013).

O questionário foi adaptado em formulário virtual, onde os estudantes leram e concordaram com o Termo de Consentimento Livre e Esclarecido da pesquisa, e participaram da mesma de forma anônima. Os estudantes que concordaram em participar do estudo tiveram que responder a uma única pergunta que questionava sobre os impactos negativos do ensino remoto, visando identificar quais as principais dificuldades do ensino remoto e uso das tecnologias vivenciados e/ou percebidos pelos mesmos durante o modelo remoto de ensino. A questão foi uma pergunta aberta, assim, não houve delimitação das alternativas de resposta, permitindo que os discentes respondessem com total liberdade seus pontos de vista e percepçóes sobre suas vivências.

Foi feita uma análise de conteúdo das respostas para categorização das mesmas em diferentes fatores negativos do ensino remoto. Para tal foi usada a técnica de análise de conteúdo, que permite o exame de dados escritos e semelhantes com a finalidade de realizar uma leitura crítica e aprofundada para permitir interpretações e inferências através de etapas de pré-análise, descrição analítica do material e interpretação dos resultados (da Silva, de Oliveira, Brito, 2021). Também foram utilizadas referências como bases metodológicas e epistemológicas para conhecimento e interpretação do processo de ensino (de França, 2003). Os dados quantitativos sobre cada categoria de resposta identificada foram inseridos e analisados no Software Microsoft Excel ${ }^{\circ}$ e expressos em frequência absoluta e em frequência relativa.

\section{Resultados E Discussão}

A análise de conteúdo das respostas dos 26 estudantes permitiu a categorização das respostas em cinco fatores, que correspondem às principais dificuldades apontadas pelos estudantes no ensino-aprendizagem de Educação Física, durante a pandemia da COVID-19, com a aplicação do modelo remoto de ensino e uso das tecnologias para viabilizá-lo (Tabela 1). 
TABELA 1

Principais dificuldades do ensino remoto e uso das tecnologias, durante a pandemia da COVID-19, no ensino-aprendizagem de Educação Física apontadas pelos estudantes

\begin{tabular}{cc}
\hline Dificuldades apontadas & $\begin{array}{c}\text { Frequência absoluta das respostas (\% dos } \\
\text { estudantes) }\end{array}$ \\
\hline Metodologias de avaliação insatisfatórias & $12(46,15 \%)$ \\
Problemas com o acesso à internet e recursos & $9(34,61 \%)$ \\
tecnológicos & $9(34,61 \%)$ \\
Prejuículdades de aprendizagem & $7(26,92 \%)$ \\
Ausência de aulas práticas e aumento do & $2(7,69 \%)$ \\
comportamento sedentário & \\
\hline
\end{tabular}

Fonte: Os autores (2021).

Aproximadamente metade dos estudantes avaliados concorda que as metodologias de avaliação no ensino remoto são insatisfatórias, correspondendo à uma alta demanda de atividades e ao emprego inadequado ou ausente das tecnologias para uma avaliação mais eficiente. Tal fator é justificado pelo processo adaptativo ainda precário e insuficiente dos professores à nova realidade de ensino, onde os mesmos não possuem domínio no desenvolvimento de dinâmicas e atividades facilitadoras através de tecnologias, sendo a alta demanda de atividades no ensino remoto durante a pandemia da COVID-19 uma complicação apontada por diversos estudos (Andreza et al., 2020).

Para reverter este aspecto diante da implementação do modelo remoto de ensino, além de evitar excesso de atividades, os docentes de Educação Física bem como os de outras áreas do conhecimento precisam diversificar os instrumentos de avaliação, realizando-as não somente ao final das disciplinas mas durante o desenvolvimento da mesma, promovendo também trabalhos em coletivo e não apenas individuais, adotando avaliações baseadas na criatividade, reflexão, autonomia e criticidade dos estudantes (Nakashima, 2020).

Outra parte considerável dos estudantes destacou os problemas com o acesso à internet e recursos tecnológicos, evidenciando desde problemas quanto às conexões para navegação na internet e participação das aulas por videochamadas, quanto à exclusão de estudantes mais carentes. Embora atualmente estamos vivendo um cenário cada vez mais de promoção à inserção das tecnologias de informação e comunicação no ambiente acadêmico, existem dificuldades inerentes a interação on-line na educação, porque uma parte da população ainda não tem acesso à internet e a equipamentos tecnológicos, tais como notebook, tablet e computador de mesa, o que dificulta a realização das atividades e os encontros virtuais em tempo real entre alunos e professores (Arruda \& Siqueira, 2021).

Cardoso, Ferreira \& Barbosa (2020) complementam a discussão dos nossos resultados quanto à problemas com acesso à internet e recursos tecnológicos pelos estudantes ao esclarecerem que os obstáculos à igualdade e ao acesso à educação no período remoto por acadêmicos brasileiros durante o isolamento social não são as aulas ministradas virtualmente com aparatos tecnológicos em si, e sim a ausência de um plano nacional de ação educacional de emergência estruturado e articulado para alcançar todos os alunos, sem exceção, capaz de promover à população inclusão na condução do processo educacional durante a pandemia, assim 
como a elaboração e aplicação de planos de enfrentamento e superação da crise causada pelo coronavírus que inevitavelmente atingirá a educação.

Em relação às dificuldades de aprendizagem, 34,61\% dos estudantes apontaram sofrer com a falta de atenção e motivação. Segundo eles, aspectos que incluem a falta de encontro com os colegas e professores, a desmotivação para habilitar a câmera durante as aulas e as dificuldades em conciliar as aulas com o ambiente domiciliar são os principais fatores que acabam repercutindo negativamente sobre a aprendizagem. Tais opinióes evidenciam a interferência dos efeitos psicológicos do isolamento social na aprendizagem dos estudantes durante o ensino remoto, aspecto também observado por Vieira et al. (2020) ao identificarem que grande parte dos estudantes universitários analisados sofreram de alteraçóes de humor e sentimentos de angústia e ansiedade no ensino remoto durante a pandemia da COVID-19, o que afetou a produtividade dos estudantes. Além das dificuldades de equilíbrio emocional, a falta de um ambiente tranquilo em casa para estudar à distância e se dedicar aos estudos, bem como a falta de tempo para estudar por conta das atividades do próprio lar, já são apontados na literatura como os principais desafios do jovens brasileiros para estudar em casa durante o ensino remoto (dos Santos Silva \& Peixoto, 2020).

É provável que a desmotivação apresentada também tenha relação com a diferença de metodologias apresentadas entre as aulas presenciais e remotas, ao que tudo indica, as aulas realizadas no formato presencial parecem despertar um maior interesse por parte dos estudantes (Deng et al., 2020; Dost et al., 2020). Quando observamos a literatura, vemos que essas dificuldades não se restringem apenas a estudantes brasileiros. Um estudo conduzido em Wuhan na China, com mais de 1.600 estudantes da graduação do curso de Educação Física apontou que a realização das aulas de forma remota não foi considerada satisfatória por mais de 50\% dos alunos entrevistados (Deng et al., 2020).

Quanto aos prejuízos na qualidade do ensino, os estudantes de nosso estudo que citaram esta dificuldade falaram sobre o fato de muitos professores não terem qualificação necessária para uso de tecnologias no ensino remoto, trabalhando com uma metodologia pedagógica fraca no cenário de educação pandêmico e de contexto de isolamento social. Tais dificuldades encontradas podem ser explicadas, como apontam Oliveira, Silva \& Silva (2020), pelo fato de muitos professores estarem tendo o primeiro contato ativo com o meio virtual de ensino neste período emergencial em face da pandemia da COVID-19, tendo que lidar de forma abrupta com novas formas de ensinar e de aprender por meio das tecnologias digitais sem ter formação prévia ou mesmo ambientação às redes virtuais. Os mesmos autores discutem a necessidade de reconfiguração do ensino utilizando-se as tecnologias, onde os professores são capazes de ampliar e criar práticas pedagógicas que potencializem as interações no processo de ensino-aprendizagem, contribuindo com o desenvolvimento intelectual e cultural dos alunos, bem como com uma nova forma de concepção de currículo e organização escolar.

Resultados parecidos foram identificado em pesquisa similar, onde a maioria dos acadêmicos informou que o uso de metodologias ativas no ensino remoto pelos professores não é frequente, sendo então um dado preocupante considerando que para que o ensino remoto tenha qualidade as aulas precisam ser bem elaboradas e dinâmicas (Neto et al., 2021), evitando a metodologia tradicional de ensino. Recomenda-se, então, o uso de metodologias ativas no ensino de Educação Física aliadas às tecnologias pois elas promovem função autônoma e crítica aos estudantes, onde eles são o centro do processo ensino-aprendizagem e o professor condutor da aula, estimulando, assim, o desenvolvimento dos discentes e suas compreensões políticas, culturais, afetivas, cognitivas e socioeconômicas (Vieira \& Santos, 2020).

Por fim, uma pequena parcela dos estudantes pontuou como aspecto negativo do ensino remoto e uso de tecnologias no ensino-aprendizagem da Educação Física, durante a pandemia da COVID-19, a ausência de aulas práticas e promoção do aumento do comportamento sedentário. Durante a pandemia a rotina de estudos e trabalho precisou ser ajustado de forma remota, o que favoreceu a maior prevalência de inatividade física e comportamentos sedentários em brasileiros (Silva et al., 2021a; Werneck et al., 2021), assim como a faixa etária mais jovem parece apresentar maior incidência de inatividade física e uso aumentado 
de computador, sendo concluído que as pessoas que aderiram às medidas mais rígidas de quarentena apresentaram maior incidência de tempo excessivo de tela (Silva et al., 20201b; Werneck et al., 2021).

Este aspecto do aumento do comportamento sedentário é alarmante, como mostra um estudo em países ocidentais que mediu dimensões planejadas e não planejadas de atividade física em estudantes, durante o período de isolamento social pela pandemia da COVID-19, usando o Questionário de Atividade Física de Estilo de Vida de Brunel e a saúde mental usando o Questionário de Saúde Geral de 12 itens. Foram observados prevalência de comportamento sedentário e efeitos deletérios da inatividade física e saúde mental nos estudantes de todos os países investigados (Karageorghis et al., 2021).

Cada vez mais as pesquisas sugerem incluir a atividade física como possibilidade, estratégia ou práticas de combater o comportamento sedentário em ambientes onde se espera que os indivíduos fiquem sentados a maior parte do tempo, como mostra o estudo de Pilcher et al. (2017). Na pesquisa, estudantes universitários foram submetidos a um protocolo em cicloergômetro enquanto estudavam, e os resultados indicaram que estações de trabalho de atividade física podem ser implementadas para incentivar a atividade física leve, sem afetar negativamente o desempenho acadêmico, proporcionando benefícios de saúde e bemestar a longo prazo. Assim, o ajuste atual para aulas remotas parece dificultar e aumentar o tempo em comportamento sedentário, sendo necessário então que os professores de Educação Física desenvolvam aulas práticas individuais que possam ser realizadas no espaço domiciliar dos estudantes, bem como que o sistema educacional desenvolva iniciativas para a quebra do comportamento sedentário durante as aulas remotas, beneficiando a saúde física e mental.

Como limitação da pesquisa, podemos mencionar o número amostral pequeno em relação à população de estudantes do curso de Educação Física que formam o corpo acadêmico da instituição do estudo, entretanto a análise e identificação dos principais problemas envolvidos ao ensino-aprendizagem na graduação em Educação Física durante a primeira implementação do ensino-remoto em face à pandemia da COVID-19 que puderam ser discutidos no estudo são de grande valia para verificar necessidades e propor soluções para os problemas educacionais debatidos.

\section{CONSIDERAÇÕES FINAIS}

Conclui-se que, para os estudantes de Educação Física investigados, as principais dificuldades do ensino remoto e uso de tecnologias durante a pandemia da COVID-19 no ensino-remoto são as metodologias de avaliação insatisfatórias, problemas com o acesso à internet e recursos tecnológicos, algumas dificuldades de aprendizagem e prejuízos na qualidade do ensino, e por fim a ausência de aulas práticas e o aumento do comportamento sedentário.

Trazemos como direcionamentos que o ensino de Educação Física durante o ensino remoto seja planejado de forma a amenizar tais problemas identificados. Recomenda-se a formação continuada de professores sobre o uso de tecnologias na Educação Física e o trabalho com metodologias ativas retratando temas do cotidiano para facilitar a aprendizagem dos alunos e aproximá-los da discussão crítica do conhecimento, fazendo uso de sites digitais e aplicativos dinâmicos, a exemplo, evitando atividades individuais em excesso e a manutenção da perspectiva tradicional de ensino-aprendizagem. Também foi vista a necessidade de elaboração e consolidação de propostas para acessibilidade de tecnologias e acesso à internet para os estudantes, visto a discrepância social existente no ensino superior brasileiro, para evitar a exclusão e a desistência de estudantes carentes.

Para melhores condições de ensino e aprendizagem na área da Educação Física durante o período de isolamento social, sugerimos que os professores incluam em seus planos de ensino atividades de exercícios em casa e até mesmo o planejamento de atividades para serem feitas durante pausas entre as aulas, diminuindo o tempo em comportamento sedentário dos estudantes. Como principal saída para atenuar os dados na formação dos estudantes em Educação Física em relação ao ensino e aprendizagem sobre movimento e prática 
corporal, enaltecemos a possibilidade de as instituições aderirem ao ensino híbrido no cenário pandêmico e pôs-pandêmico ao passo que as condições sanitárias estiverem mais controladas. Tal medida facilitará a promoção do conhecimento e a interação entre professores e estudantes, bem como entre os próprios estudantes em si, e provavelmente também reduzirá os sintomas psicológicos negativos em decorrência do isolamento social na comunidade acadêmica.

\section{REFERÊNCIAS}

Alves, J. C. R., \& Ferreira, M. B. (2020). Covid-19: reflexão da atuação do enfermeiro no combate ao desconhecido. Enfermagem em Foco, 11(1. ESP).

Andreza, R. S., de Souza Alvesb, E. J., Martinsb, L. H., Silvab, R. H., da Silvab, S. D. A., Nogueirab, T. L., Gomes, R. N., \& da Silva Santosb, J. C. (2020). Os Impactos da Covid-19 na Educação por Meio do Ensino Remoto. Revista Interfaces, 8(3).

Aquino, E. M., Silveira, I. H., Pescarini, J. M., Aquino, R., Souza-Filho, J. A. D., Rocha, A. D. S., Ferreira A., Victor A., Teixeira C., Machado D. B., Paixão E., Alves F. J. O., Pilecco, F., Menezes G., Gabrielli L., Leite L., de Almeida M. C. C., Ortelan N., Fernandes Q. H. R. F. F., Ortiz R. J. F., Palmeira R. N., Junior E. P. P., Aragão E., de Souza L. E. P. F., Netto M. B., Teixeira M. G., Barreto M. L., Ichihara M. Y., \& Lima, R. T. D. R. S. (2020). Medidas de distanciamento social no controle da pandemia de COVID-19: potenciais impactos e desafios no Brasil. Ciência \& Saúde Coletiva, 25, 2423-2446.

Arruda, J. S., \& de Castro Siqueira, L. M. R. (2021). Metodologias Ativas, Ensino Híbrido e os Artefatos Digitais: sala de aula em tempos de pandemia. Práticas Educativas, Memórias e Oralidades-Rev. Pemo, 3(1), e314292.

Cardoso, C. A., Ferreira, V. A., \& Barbosa, F. C. G. (2020). (Des) igualdade de acesso à educação em tempos de pandemia: uma análise do acesso às tecnologias e das alternativas de ensino remoto. Revista Com Censo: Estudos Educacionais do Distrito Federal, 7(3), 38-46.

Có, E. P., Amorim, G. B., \& Finardi, K. R. (2020). Ensino de línguas em tempos de pandemia: experiências com tecnologias em ambientes virtuais. Revista Docência e Cibercultura, 4(3), 112-140.

Creswell, J. W. (2010). Projeto de pesquisa: Métodos qualitativo, quantitativo e misto. Porto Alegre: Artmed.

da Silva, B. A., de Oliveira, G. S., \& Brito, A. P. G. (2021). Análise de conteúdo: uma perspectiva metodológica qualitativa no âmbito da pesquisa em educação. Cadernos da FUCAMP, 20(44).

de França, T. L. (2003). Educação-corporeidade-lazer: Saber da Experiência Cultural em Prelúdio. Tese de Doutorado, Universidade Federal do Rio Grande do Norte, Natal, RN, Brasil.

de França Filho, A. L., da França Antunes, C., \& Couto, M. A. C. (2020). Alguns apontamentos para uma crítica da EAD na educação brasileira em tempos de pandemia. Revista Tamoios, 16(1).

Deng, C. H., Wang, J. Q., Zhu, L. M., Liu, H. W., Guo, Y., Peng, X. H., Shao J. B., \& Xia, W. (2020). Association of web-based physical education with mental health of college students in Wuhan during the COVID-19 outbreak: cross-sectional survey study. Journal of medical Internet research, 22(10), e21301.

dos Santos Silva, F. C., \& Peixoto, G. T. B. (2020). Percepção dos professores da rede estadual do Município de São João da Barra-RJ sobre o uso do Google Classroom no ensino remoto emergencial. Research, Society and Development, 9(10), e5729109023.

Dost, S., Hossain, A., Shehab, M., Abdelwahed, A., \& Al-Nusair, L. (2020). Perceptions of medical students towards online teaching during the COVID-19 pandemic: a national cross-sectional survey of 2721 UK medical students. BMJopen, $10(11)$, e042378.

Fernando, A. (2017). As tecnologias nas aulas de Educação Física Escolar. Revista Educação Pública, 17 (1). Recuperado em https://educacaopublica.cecierj.edu.br/artigos/17/1/as-tecnologias-nas-aulas-de-educao-fsica-escolar.

Godoi, M., Kawashima, L. B., de Almeida Gomes, L., \& Caneva, C. (2021). As práticas do ensino remoto emergencial de Educação Física em escolas públicas durante a pandemia da Covid-19: reinvenção e desigualdade. Revista Prática Docente, 6(1), e012. 
Hodges C., Moore S., Lockee B., Trust T., \& Bond, A. (2020, março 27). The Difference Between Emergency Remote Teaching and Online Learning. Recuperado em https://er.educause.edu/articles/2020/3/the-difference-betwe en-emergency-remote-teaching-and-online-learning\#fn3.

Karageorghis, C. I., Bird, J. M., Hutchinson, J. C., Hamer, M., Delevoye-Turrell, Y. N., Guérin, S. M., Mullin, E. M., Mellano, K. T., Parsons-Smith, R. L., Terry V. R. \& Terry, P. C. (2021). Physical activity and mental well-being under COVID-19 lockdown: a cross-sectional multination study. BMC public health, 21(1), 1-13.

Nakashima, R. H. R. (2020). Algumas considerações didático-pedagógicas sobre o ensino remoto. Participativa: Ciência Aberta em Revista, 2(1), 1-6.

Neto, B. F., Costa, J., dos Santos, M. C., dos Santos, C. E. C., Neto, G. T., dos Santos Nogueira, M., \& do Egito, R. R. (2021). A percepção dos discentes em relação aos processos de ensino e aprendizagem no período remoto em meio a pandemia. Brazilian Journal of Development, 7(5), 52013-52031.

Oliveira, S. S., Silva, O. S. F., \& Silva, M. J. O. (2020). Educar na incerteza e na urgência: implicações do ensino remoto ao fazer docente e a reinvenção da sala de aula. Interfaces Cientificas-Educação, 10(1), 25-40.

Pilcher, J. J., Morris, D. M., Bryant, S. A., Merritt, P. A., \& Feigl, H. B. (2017). Decreasing sedentary behavior: Effects on academic performance, meta-cognition, and sleep. Frontiers in neuroscience, 11, 219.

Sampieri, R. H., Collado, C. F., Lucio, M. P. B. (2013). Metodologia de pesquisa. Porto Alegre: Penso.

Silva, D. R., Werneck, A. O., Malta, D. C., Souza-Júnior, P. R., Azevedo, L. O., Barros, M. B., \& Szwarcwald, C. L. (2021a). Incidence of physical inactivity and excessive screen time during the first wave of the COVID-19 pandemic in Brazil: what are the most affected population groups?: Impact of COVID-19 pandemic on physical activity and screen time in Brazil. Annals of epidemiology, 62.

Silva, D. R. P. D., Werneck, A. O., Malta, D. C., Souza Júnior, P. R. B. D., Azevedo, L. O., Barros, M. B. D. A., \& Szwarcwald, C. L. (2021b). Changes in the prevalence of physical inactivity and sedentary behavior during COVID-19 pandemic: a survey with 39,693 Brazilian adults. Cadernos de Saúde Pública, 37, e00221920.

Valente, G. S. C., de Moraes, É. B., Sanchez, M. C. O., de Souza, D. F., \& Pacheco, M. C. M. D. (2020). O ensino remoto frente às exigências do contexto de pandemia: Reflexões sobre a prática docente. Research, Society and Development, 9(9), e843998153.

Vieira, T. D. G. F., \& dos Santos, M. L. S. C. (2020). Estratégias pedagógicas e uso de metodologias ativas na graduação em Enfermagem em tempos de pandemia do Coronavírus-COVID-19. Research, Society and Development, 9(11), e2759119749.

Vieira, K. M., Postiglioni, G. F., Donaduzzi, G., dos Santos Porto, C., \& Klein, L. L. (2020). Vida de estudante durante a pandemia: isolamento social, ensino remoto e satisfação com a vida. EaD em Foco, 10(3).

Werneck, A. O., Silva, D. R., Malta, D. C., Souza-Júnior, P. R., Azevedo, L. O., Barros, M. B., \& Szwarcwald, C. L. (2021). Changes in the clustering of unhealthy movement behaviors during the COVID-19 quarantine and the association with mental health indicators among Brazilian adults. Translational behavioral medicine, 11(2), 323-331. 